\title{
Comparison of speciation and bioavailability of rare earth elements between wet rhizosphere soil and air-dried bulk soil
}

\author{
Zhongwen Wang, Xiao-quan Shan*, Shuzhen Zhang \\ Research Center for Eco-Environmental Sciences, Chinese Academy of Sciences, P.O. Box 2871, Beijing 100085, China
}

Received 12 December 2000; received in revised form 15 March 2001; accepted 4 May 2001

\begin{abstract}
A comparison was made between the speciation and bioavailability of rare earth elements in rhizosphere and nonrhizosphere soils and between wet and air-dried soils under greenhouse conditions by using a homemade rhizobox. The speciation of rare earth elements in soils is experimentally defined as water soluble, exchangeable and carbonate bound (B1), Fe-Mn oxide bound (B2) and organic matter and sulfide bound (B3). The air-drying process of the wet soils resulted in an increase in rare earth elements in fractions B1 and B2, and a decrease in B3. Generally, a better correlation was obtained between the speciation and bioavailability by using wet soils than that by using air-dried soils, and as the conditions in the former are close to the realistic conditions of plant growth in the environment better plant availability is also achieved between the speciation of rare earth elements in the rhizosphere soils than that in the nonrhizosphere soils. Application of the rhizosphere wet soils is be strongly recommended in the future study on the speciation analysis and bioavailability of trace elements in soils. (C) 2001 Elsevier Science B.V. All rights reserved.
\end{abstract}

Keywords: Speciation; Bioavailability; Accumulation; Rhizosphere; Air-drying; Rare earth elements

\section{Introduction}

Over the past decades rare earth elements have been widely applied in industry and agriculture. Particularly, millions of tons of fertilizers containing rare earth elements are used worldwide due to the increase in agricultural productivity. Since early 1990s such fertilizers have been applied in China and were estimated to cover approximately $3.7 \times 10^{6}$ ha in 1993 and 1.6-2.0 $\times 10^{7}$ ha in $1995[1,2]$. Therefore, more and more rare earth elements enter the environment through these human activities, and thus, may disturb the elemental balances in the environment. Despite the

\footnotetext{
* Corresponding author. Tel.: +86-10-62923560; fax: +86-10-62923563.

E-mail address: xiaoquan@mail.rcees.ac.cn (X.-q. Shan).
}

reported increase in agriculture yields by application of these fertilizers the possible long-term hazardous environmental effects are worth investigating comprehensively. There are several reports on the toxic effects of rare earth elements and ill-effects due to occupational and environmental exposure to rare earth elements [3-6]. Many efforts have been made to understand the distribution and accumulation of rare earth elements in the environment. Wyttembach et al. [7] studied the behavior of rare earth elements in soils and plants in Switzerland. Ichihashi et al. [8] investigated the background level of rare earth elements in various environmental media and biota in Japan. Diatoff et al. [9] and Tu et al. [10] reported the accumulation of rare earth elements in soil and carp, respectively. Nowadays, people are aware of the lesser importance of total concentrations of rare earth elements 
in samples because the total concentrations are not directly related to the potential interactions of rare earth elements with the environment. Therefore, it is important to obtain information of elemental species present in samples.

Bryan and Langston [11] comprehensively reviewed the progress in the bioavailability, accumulation and effects of heavy metals with emphasis on sediments. Generally, experimentally defined single and/or multiple sequential extraction procedures are used to obtain the elemental fractionations, and then a single or multiple regression analysis is performed to obtain the correlations between the speciation of the elements of interests in soils and the concentrations of those elements in plants. The goodness of the correlation coefficients determines the bioavailability. The most widely used sequential extraction methods are those recommended by Tessier et al. [12] and the Community Bureau of Reference (BCR) [13]. Although these methods suffer from the nonselectivity of extractants used and redistribution of trace elements among the solid phases during the extraction processes [14,15], they are still widely used to study the speciation and bioavailability of rare earth elements because there are no better methods available [16-18].

It should be pointed out that plants may exude many low molecular weight organic acids, which make the rhizosphere a small but important microenvironment, and where the physicochemical behavior and uptake processes of elements by plants should be quite different from bulk soil $[19,20]$. To our knowledge this is the first report on the speciation and bioavailability of rare earth elements in the rhizosphere. The aim of the present study is to compare the speciation and bioavailability of rare earth elements between the rhizosphere and nonrhizosphere soils. The effect of air-drying of soil sample on the speciation and bioavailability is also discussed.

\section{Materials and methods}

\subsection{Soils}

Soil samples were collected from 15 rural area around China, representing typical Chinese soils with different physical and chemical properties. All soils were taken from the surface layer $(0-20 \mathrm{~cm})$ of cultivated soils. The soils were air-dried, ground and sieved
Table 1

Selected properties of the studied soils

\begin{tabular}{|c|c|c|c|c|}
\hline Sample & Site & $\mathrm{pH}$ & $\begin{array}{l}\text { CEC } \\
\left(\mathrm{cmol} \mathrm{kg}^{-1}\right)^{\mathrm{a}}\end{array}$ & $\mathrm{OM}(\%)^{\mathrm{b}}$ \\
\hline 1 & Jiamusi & 5.97 & 27.03 & 4.30 \\
\hline 2 & Lanzhou & 7.43 & 10.68 & 2.53 \\
\hline 3 & Shaanxi & 8.25 & 25.88 & 1.75 \\
\hline 4 & Shanxi & 7.92 & 21.86 & 4.12 \\
\hline 5 & Yuxi & 4.45 & 25.78 & 1.37 \\
\hline 6 & Kunming & 5.55 & 26.74 & 4.22 \\
\hline 7 & Shandong & 5.94 & 30.20 & 3.62 \\
\hline 8 & Jiangxi & 5.49 & 14.24 & 1.53 \\
\hline 9 & Shanghai & 5.53 & 36.65 & 3.02 \\
\hline 10 & Hangzhou & 7.05 & 19.76 & 3.28 \\
\hline 11 & Henan & 7.73 & 18.12 & 2.03 \\
\hline 12 & Wuhan & 7.27 & 53.98 & 2.37 \\
\hline 13 & Changsha & 5.50 & 43.11 & 2.28 \\
\hline 14 & Hefei & 6.71 & 24.95 & 2.09 \\
\hline 15 & Dongguan & 7.45 & 15.92 & 1.37 \\
\hline
\end{tabular}

${ }^{\text {a }} \mathrm{CEC}$ : cation exchange capacity.

${ }^{\mathrm{b}} \mathrm{OM}$ : organic matter.

to pass through a $1 \mathrm{~mm}$ plastic mesh for further use. Precautions were taken to avoid contamination during sampling, drying, grinding and storage. Soil properties of $\mathrm{pH}$, organic matter $(\mathrm{OM})$ and cation exchange capacity (CEC) were determined using standard methods [21]. These properties are given in Table 1 .

\subsection{Greenhouse experiment}

A homemade rhizobox (Fig. 1) [22] was used to plant wheat. The dimension of the rhizobox was $120 \mathrm{~mm} \times 100 \mathrm{~mm} \times 150 \mathrm{~mm}$ (length $\times$ width $\times$ height $)$. The rhizobox was divided into three sections: a central zone or rhizosphere zone ( $20 \mathrm{~mm}$ in length), which was surround by nylon cloth (300 mesh), and left and right nonrhizosphere zones $(50 \mathrm{~mm}$ in length). Each nonrhizosphere soil was further evenly separated into three subzones by nylon cloth, which are referred to as near rhizosphere zone, near bulk soil zone and bulk soil zone according to the distance from the rhizosphere zone to the boundary of the rhizobox. The previously air-dried soils were treated with $0.4 \mathrm{~g} \mathrm{~kg}^{-1} \mathrm{~N}$ and $\mathrm{P}$, as a solution of $\mathrm{KH}_{2} \mathrm{PO}_{4}$ and $\left(\mathrm{NH}_{4}\right)_{2} \mathrm{CO}_{3}$. After the soil and solution were mixed thoroughly, the soils were air-dried, and ground to pass a $1 \mathrm{~mm}$ mesh sieve. To each rhizobox was added $1.5 \mathrm{~kg}$ of the treated soil. Soils were not sterilized. 


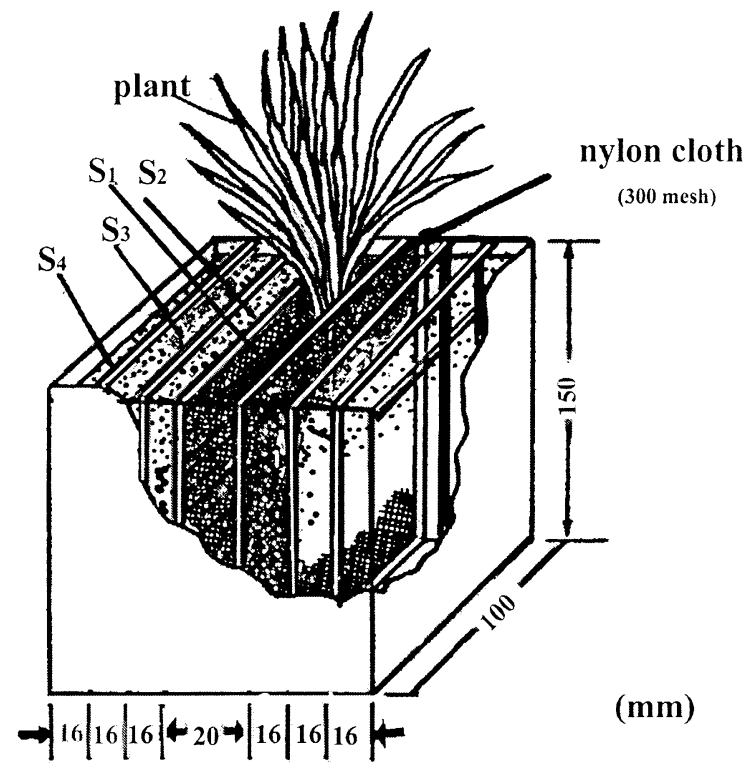

Fig. 1. Sketch of a rhizobox.

Wheat seeds (Troticum aestivum L.) were thoroughly rinsed with water, and germinated on a filter paper. After the wheat seeds were germinated for $24 \mathrm{~h}$ at $20^{\circ} \mathrm{C}$ in the dark, uniformly germinated seeds with radical emerged were sown in the soil, which had been left in the rhizobox to equilibrate overnight. A total of 30 seeds were sown per rhizosphere zone and subsequently thinned to 20 plants. Plants were grown under greenhouse conditions. The temperature ranged from 20 to $25^{\circ} \mathrm{C}$ during the day and 10 to $15^{\circ} \mathrm{C}$ during the night. The relative humidity was kept around $15 \%$ by adding deionized water daily.

Plants were harvested 8 weeks after germination. The aboveground parts of the plants were first harvested as shoots. The rhizobox was then dismantled. The roots were found completely in the central zone, while the mycelium was found to have perforated the nylon cloth. Roots were washed out with tap water to remove soil. Both the roots and shoots were rinsed with deionized water, then dried at $60^{\circ} \mathrm{C}$ for $48 \mathrm{~h}$, and the dried sample was finely ground for analysis.

\subsection{Speciation analysis}

Rhizosphere soil was sieved gently to remove the roots by keeping the root mass intact as much as possible. Rootlets that passed through the sieve were subsequently removed with a pair of tweezers. The soils taken from two near rhizosphere soil zones, a near bulk soil zone, and a bulk soil zone of the left and right were mixed separately for further speciation analysis. The four soil samples collected from the rhizosphere, near rhizosphere, near bulk soil and bulk soil zones were designated as S1, S2, S3 and S4, respectively.

A portion of $1.000 \mathrm{~g}$ of the wet samples $\mathrm{S} 1, \mathrm{~S} 2$, S3, S4 were weighed immediately after sampling and homogenizing and put into $50 \mathrm{ml}$ polypropylene centrifuge tubes for fraction analysis with a three-stage sequential extraction procedure proposed by BCR [13]. They were specified as fraction B1: water soluble, exchangeable and carbonate bound; fraction B2: Fe-Mn oxide bound; and fraction B3: organic matter and sulfide bound. At the same time, subsamples were taken to determine the soil moisture content. A portion of soils S1 and S4 was air-dried, ground and passed through a $1 \mathrm{~mm}$ mesh. The air-dried samples were extracted by the same speciation analysis procedure as the wet sample except only $0.5000 \mathrm{~g}$ of dried soil was used. All the speciation analysis was performed in duplicate and the average values were used for further analysis.

\subsection{Determination of rare earth elements}

Total contents of $\mathrm{La}, \mathrm{Ce}, \mathrm{Pr}$ and $\mathrm{Nd}$ in plant and soil were determined by inductively coupled plasma-mass spectrometry (ICP-MS) (VG PlasmaQuard 3, Fisons Instruments, UK) after samples were decomposed with $3 \mathrm{ml}$ of a $\mathrm{HNO}_{3}-\mathrm{HF}-\mathrm{HClO}_{4}$ mixture $(1: 1: 1, \mathrm{v} / \mathrm{v})$ under high pressure conditions [23]. The ranges and mean values are presented in Table 2. The rare earth elements in each fraction were also determined by ICP-MS. The optimal operating conditions for the determinations were reported previously [16].

\subsection{Reagent and statistics}

All reagents used were of analytical reagent grade or better. Correlation coefficients were estimated with the linear regression program in the statistical software of Microcal_origin _(Microcal Software, Inc, Northampton, MA 01060). 
Table 2

Total concentrations of $\mathrm{La}, \mathrm{Ce}, \mathrm{Pr}$ and $\mathrm{Nd}$ in soils, shoots and roots $\left(\mu \mathrm{gg}^{-1}\right)$

\begin{tabular}{|c|c|c|c|c|c|c|}
\hline & \multicolumn{2}{|l|}{ Soil } & \multicolumn{2}{|l|}{ Root } & \multicolumn{2}{|l|}{ Shoot } \\
\hline & Range & Mean & Range & Mean & Range & Mean \\
\hline $\mathrm{La}$ & $13.25-41.05$ & 24.20 & $0.73-8.96$ & 4.30 & $0.053-0.22$ & 0.11 \\
\hline $\mathrm{Ce}$ & $29.45-87.45$ & 50.98 & $4.96-16.5$ & 11.29 & $0.87-0.51$ & 0.19 \\
\hline $\operatorname{Pr}$ & $3.06-9.89$ & 5.62 & $0.17-1.81$ & 1.03 & $0.01-0.06$ & 0.02 \\
\hline $\mathrm{Nd}$ & $10.75-36.15$ & 19.78 & $0.58-5.57$ & 3.53 & $0.03-0.22$ & 0.06 \\
\hline
\end{tabular}

\section{Results and discussion}

\subsection{Accumulation of rare earth elements in wheat}

Although the 14 rare earth elements are recognized as a whole group, generally, they are divided into three parts: the light ( $\mathrm{La}$ to $\mathrm{Eu}$ ), middle ( $\mathrm{Sm}$ to Ho), and heavy rare earth elements ( $\mathrm{Gd}$ to $\mathrm{Lu})$. In this paper, only $\mathrm{La}, \mathrm{Ce}, \mathrm{Pr}$ and $\mathrm{Nd}$ were investigated as they represent most of the rare earth resources in the environment and are the main component in the rare earth fertilizer as well $[7,24]$. The range and mean values of $\mathrm{La}, \mathrm{Ce}, \mathrm{Pr}$ and $\mathrm{Nd}$ in shoots and roots are given in Table 2. Apparently, the concentrations of $\mathrm{La}, \mathrm{Ce}, \mathrm{Pr}$ and $\mathrm{Nd}$ in the root, ranging from 1.03 to $4.30 \mu \mathrm{g} \mathrm{g}^{-1}$, are higher than those in the shoot, in which the mean value is from 0.02 to $0.11 \mu \mathrm{g} \mathrm{g}^{-1}$. For different rare earth elements, the accumulation trend is different. Using their mean concentration in soils as the background value, the ratio of mean values in root to that in soil for $\mathrm{La}, \mathrm{Ce}, \mathrm{Pr}$ and $\mathrm{Nd}$ is 17.75 , $22.09,18.40$ and $17.80 \%$, respectively. The concentration sequence in the root is similar to their relative abundance sequence in soil. This phenomenon reveals that the root accumulates rare earth elements from soils without discrimination. However, the ratio of mean concentration of rare earth elements in shoots to that in soil was found to be $0.45,0.39,0.37$ and $0.35 \%$, respectively, which revealed that the concentration order in shoots was slightly different from that in roots. It also clarified that La would be easily uptaken by shoots. The physiological processes related to the uptake of rare earth elements by plant are very complicated [25], and there may be some special mechanism responsible for the selective transport of La from root to shoot in wheat. However, no experimental evidence is available at the present time.

\subsection{Speciation difference between air-dried and wet soil samples}

Speciation differences caused by air-drying were reported for heavy metals and radioactive elements. However, the possible influence of air-drying on the elemental speciation of rare earth elements has not been reported.

Both the wet and air-dried rhizosphere soil (S1) and bulk soil (S4) were subjected to speciation analysis with the BCR sequential extraction procedure and the distribution of $\mathrm{La}, \mathrm{Ce}, \mathrm{Pr}$ and $\mathrm{Nd}$ in fractions B1, B2 and $\mathrm{B} 3$ was compared. The means and ranges of $\mathrm{La}$, $\mathrm{Ce}, \mathrm{Pr}$ and $\mathrm{Nd}$ in fractions B1, B2 and B3 by using wet and dried samples are listed in Table 3. Since a similar tendency can be found in S1 and S4 for $\mathrm{La}, \mathrm{Ce}, \mathrm{Pr}$ and $\mathrm{Nd}$, the effect of air-drying on $\mathrm{La}$ in three fractions B1, B2 and B3 of S1 are displayed graphically as an example (Fig. 2), which reveals the significant influence of air-drying on the speciation of rare earth elements. A brief discussion is given below.

\subsubsection{Fraction $B 1$}

As can clearly be seen from Fig. 2, La in fraction B1 of air-dried samples is higher than that of wet samples. Fraction B1 includes the water soluble, exchangeable and carbonate bound elements, which is the most mobile form of the elements and thus is the most easily available part. Similar results were reported in the literature for heavy metals [26,27], showing a decrease in available $\mathrm{Mn}, \mathrm{Zn}, \mathrm{Fe}$ and $\mathrm{Cu}$ with increase of soil moisture. The reason was attributed to the increased contents of soluble organic and dispersible organic C in soils when the soil was drying. Raveh and Avnimelech [28] suggested that drying might destroy the hydrogen bond that stabilized organic micelles, so that some organic matter decomposed, resulting in the increase of rare earth elements in fraction B1. 
Table 3

Mean and range of rare earth elements in fractions B1, B2 and B3 from S1 and S4 of 15 soils by using wet samples (w) and dry samples (d) $\left(\mu g^{-1}\right)$

\begin{tabular}{|c|c|c|c|c|c|c|c|c|c|}
\hline \multirow[t]{3}{*}{ Element } & \multirow[t]{3}{*}{ Fraction } & \multicolumn{4}{|l|}{$\mathrm{S} 1$} & \multicolumn{4}{|l|}{ S4 } \\
\hline & & \multicolumn{2}{|l|}{ w } & \multicolumn{2}{|l|}{ d } & \multicolumn{2}{|l|}{ w } & \multicolumn{2}{|l|}{ d } \\
\hline & & Range & Mean & Range & Mean & Range & Mean & Range & Mean \\
\hline \multirow[t]{3}{*}{$\mathrm{La}$} & B1 & $0.25-1.44$ & 0.53 & $0.29-2.10$ & 0.72 & $0.08-0.36$ & 0.21 & $0.11-0.67$ & 0.31 \\
\hline & B2 & $0.05-9.12$ & 3.11 & $0.14-11.06$ & 4.57 & $0.14-4.60$ & 1.70 & $0.18-5.86$ & 2.28 \\
\hline & B3 & $8.00-39.15$ & 20.63 & $5.76-25.21$ & 14.15 & $2.95-22.15$ & 8.42 & $2.33-11.2$ & 5.94 \\
\hline \multirow[t]{3}{*}{$\mathrm{Ce}$} & B1 & $0.37-2.35$ & 0.87 & $0.53-3.93$ & 1.28 & $0.14-0.82$ & 0.38 & $0.22-1.45$ & 0.60 \\
\hline & B2 & $0.07-16.29$ & 6.17 & $0.25-20.46$ & 9.35 & $0.18-7.91$ & 3.06 & $0.22-11.8$ & 4.48 \\
\hline & B3 & $17.80-85.65$ & 50.48 & $14.76-62.03$ & 35.62 & $6.21-46.90$ & 17.51 & $4.68-24.52$ & 13.12 \\
\hline \multirow[t]{3}{*}{$\operatorname{Pr}$} & B1 & $0.05-0.27$ & 0.10 & $0.07-0.49$ & 0.15 & $0.02-0.08$ & 0.04 & $0.02-0.94$ & 0.07 \\
\hline & B2 & $0.01-1.36$ & 0.52 & $0.03-1.74$ & 0.79 & $0.03-0.84$ & 0.31 & $0.04-1.05$ & 0.42 \\
\hline & B3 & $2.37-10.37$ & 5.64 & $1.71-6.97$ & 3.98 & $0.82-5.59$ & 2.39 & $0.67-3.30$ & 1.68 \\
\hline \multirow[t]{3}{*}{$\mathrm{Nd}$} & B1 & $0.19-1.06$ & 0.38 & $0.23-1.57$ & 0.53 & $0.07-0.31$ & 0.16 & $0.02-0.63$ & 0.25 \\
\hline & B2 & $0.03-4.70$ & 1.74 & $0.10-6.18$ & 2.64 & $0.08-2.47$ & 1.01 & $0.11-3.93$ & 1.44 \\
\hline & B3 & $9.12-41.05$ & 21.46 & $6.72-26.1$ & 15.08 & $2.93-21.23$ & 8.63 & $2.51-11.3$ & 6.34 \\
\hline
\end{tabular}

\subsubsection{Fraction $B 2$}

Air-drying also increased the concentration of rare earth elements in fraction B2. Of course, the increment is quite different for different soils and for $\mathrm{La}$, $\mathrm{Ce}, \mathrm{Pr}$ and $\mathrm{Nd}$ as well. Rapin et al. [29] have found that air-drying would facilitate the crystallization of $\mathrm{Fe}-\mathrm{Mn}$ oxides, thus, promoting the formation of new $\mathrm{Fe}-\mathrm{Mn}$ oxides, causing an increase in metals bound to the Fe-Mn oxides. This explanation also verified, at least to a certain extent, the experimental results of the present study.

\subsubsection{Fraction $B 3$}

A reverse phenomenon appeared in fraction $\mathrm{B} 3$ as compared with fractions B1 and B2. Air-drying caused a decrease of rare earth elements in fractions B3 by about 30\%. Bartlett and James [30] have suggested that during air-drying the oxidation of organic matter would occur. Combining the explanation of Raveh and Avnomelech [28] with that given by Bartlett and James [30], this phenomenon can easily be recognized.

\subsection{Comparison between speciation and bioavailability of $\mathrm{La}, \mathrm{Ce}, \mathrm{Pr}$ and $\mathrm{Nd}$ in air-dried and wet samples}

Single correlation analysis was performed between both the concentrations of rare earth elements in wheat shoots and roots with the different chemical speciation of $\mathrm{La}, \mathrm{Ce}, \mathrm{Pr}$, and $\mathrm{Nd}$ in soils. A poor correlation was obtained between the concentration of rare earth elements in roots and that in fractions B1, B2 and B3 (data not shown), while a significant correlation was found between the concentrations in the shoots and in the soil fractions. Correlation coefficients are given in Table 4. Apparently, the concentrations in fraction $\mathrm{B} 1$ have a significant correlation with $\mathrm{La}, \mathrm{Ce}, \mathrm{Pr}$ and $\mathrm{Nd}$ concentrations in the shoots. Since there is interstitial water in the rhizosphere soils and it is closer to the realistic situation of plant growing process as to wet soils, the better correlation obtained between the concentrations of rare earth elements in fraction B1 and that in plant shoot by using wet soils is expected. In addition, the shoot uptake also correlates well with fraction B2, because fraction B2 was of some significance to plant nutrient acquisition [31], but may be less bioavailable than fraction B1.

Careful analyses of the correlation coefficients in Table 4 suggest that the correlation coefficients obtained with wet samples were better than with dry samples. For instance, the correlation coefficients of fraction $\mathrm{B} 1$ of $\mathrm{La}\left(r_{\mathrm{S} 1, \mathrm{w}}=0.830^{* *}, r_{\mathrm{S} 1, \mathrm{~d}}=\right.$ $\left.0.792^{* *} ; r_{\mathrm{S} 4, \mathrm{w}}=0.461^{*}, r_{\mathrm{S} 4, \mathrm{~d}}=0.403\right)$ and $\mathrm{Ce}$ $\left(r_{\mathrm{S} 1, \mathrm{w}}=0.908^{* *}, r_{\mathrm{S} 1, \mathrm{~d}}=0.854^{* *} ; r_{\mathrm{S} 4, \mathrm{w}}=0.664^{* *}\right.$, $\left.r_{\mathrm{S} 4, \mathrm{~d}}=0.656^{* *}\right)$ showed this trend. This means that 

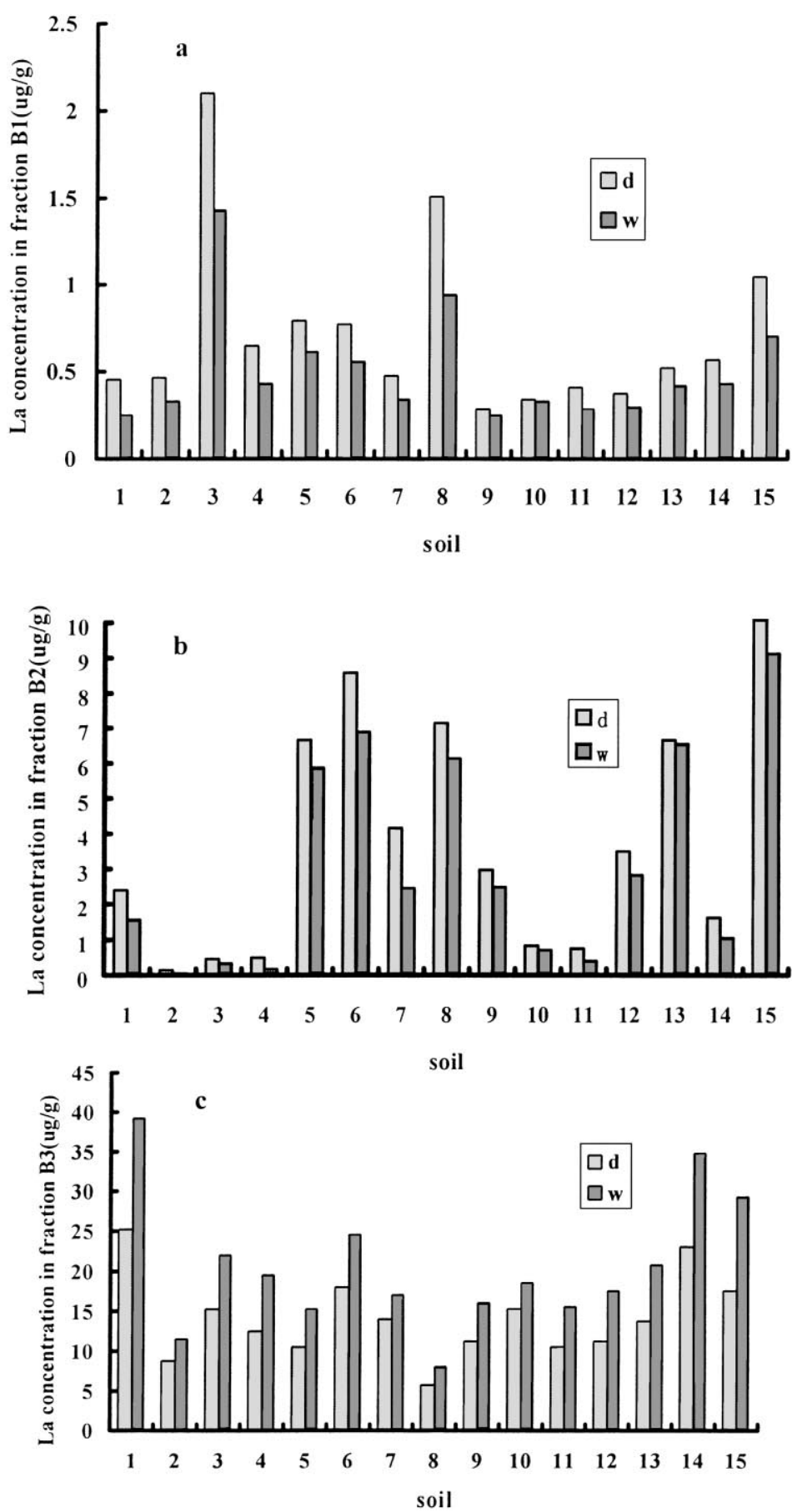

Fig. 2. Comparison of lanthanum concentration in fractions B1 (a), B2 (b) and B3 (c) between air-dried (d) and wet rhizosphere (w) soil S1. 
Table 4

Correlation coefficients for rare earth elements in shoots and soil fraction $(n=15)$

\begin{tabular}{|c|c|c|c|c|c|c|c|}
\hline \multirow[t]{2}{*}{ Element } & \multirow[t]{2}{*}{ Fraction } & \multicolumn{2}{|l|}{$\mathrm{S} 1$} & \multirow{2}{*}{$\begin{array}{l}\text { S2 } \\
\text { W }\end{array}$} & \multirow{2}{*}{$\begin{array}{l}\text { S3 } \\
\text { w }\end{array}$} & \multicolumn{2}{|l|}{ S4 } \\
\hline & & $\mathrm{d}^{\mathrm{a}}$ & $w^{b}$ & & & $\mathrm{w}$ & $\mathrm{d}$ \\
\hline \multirow[t]{3}{*}{$\mathrm{La}$} & B1 & $0.791^{* *}$ & $0.830^{* *}$ & $0.563^{* *}$ & 0.327 & $0.461^{*}$ & 0.403 \\
\hline & B2 & 0.001 & -0.027 & $0.606^{* *}$ & 0.233 & $0.437^{*}$ & 0.379 \\
\hline & B3 & -0.231 & -0.291 & -0.267 & -0.226 & -0.402 & $-0.447^{*}$ \\
\hline \multirow[t]{3}{*}{$\mathrm{Ce}$} & B1 & $0.854^{* *}$ & $0.908^{* *}$ & $0.588^{* *}$ & $0.634^{* *}$ & $0.664^{* *}$ & $0.656^{* *}$ \\
\hline & B2 & $0.794^{* *}$ & $0.806^{* *}$ & $0.725^{* *}$ & 0.186 & $0.548^{* *}$ & $0.543^{* *}$ \\
\hline & B3 & 0.290 & 0.146 & 0.121 & 0.238 & -0.134 & -0.147 \\
\hline \multirow[t]{3}{*}{$\operatorname{Pr}$} & $\mathrm{B} 1$ & $0.790^{* *}$ & $0.830^{* *}$ & 0.320 & 0.320 & $0.593^{* *}$ & 0.428 \\
\hline & B2 & $0.716^{* *}$ & $0.731^{* *}$ & $0.553^{* *}$ & 0.144 & $0.674^{* *}$ & $0.464^{* *}$ \\
\hline & B3 & 0.255 & 0.130 & 0.217 & 0.263 & -0.282 & -0.130 \\
\hline \multirow[t]{3}{*}{$\mathrm{Nd}$} & B1 & $0.790^{* *}$ & $0.854^{* *}$ & 0.334 & 0.299 & $0.629^{* *}$ & $0.431^{*}$ \\
\hline & B2 & $0.728^{* *}$ & $0.730^{* *}$ & $0.573^{* *}$ & 0.188 & $0.546^{* *}$ & 0.337 \\
\hline & B3 & 0.341 & 0.214 & 0.339 & 0.334 & -0.216 & -0.044 \\
\hline
\end{tabular}

\footnotetext{
a d: air-dried soil.

${ }^{\mathrm{b}} \mathrm{w}$ : wet soil.

* Significant at 0.10 probability level.

** Significant at 0.05 probability level.
}

the bioavailability of rare earth elements can better be expressed by using wet soil samples instead of dry-samples.

\subsection{Speciation difference among different soil zones by using wet samples}

A comparison was also made between the speciation of rare earth elements in soils obtained from the rhizosphere, near rhizosphere, near bulk soil and bulk soil by using wet soil samples. Extraction percentages of rare earth elements in four soil zones based on the total concentrations in the corresponding soils were calculated for 15 soils and are given in Fig. 3. Mean percentage values in each soil zones are used to show a speciation distribution pattern. For fraction B1, there is quite a similar concentration range from $\mathrm{S} 1$ to $\mathrm{S} 3$. However, there is much less La found in soil S4. Nevertheless, the percentage orders are $\mathrm{S} 2>\mathrm{S} 1>\mathrm{S} 3 \approx$ $\mathrm{S} 4$ and $\mathrm{S} 1>\mathrm{S} 3>\mathrm{S} 2>\mathrm{S} 4$ for fractions $\mathrm{B} 2$ and $\mathrm{B} 3$, respectively.

There is a distinct feature in Fig. 3 that the amounts of rare earth elements in fractions B1, B2 and B3 or the sum of B1, B2 and B3 was the lowest in soil zone S4 compared with other three soil zones. If the bulk soil was used the percentage of rare earth elements in $\mathrm{B} 1, \mathrm{~B} 2$ and $\mathrm{B} 3$ were generally ranged from $<1$,
$1.42-3.35$ and $7.32-30.88 \%$, respectively, compared with the total concentrations in soils [16], which was quite similar to the trends obtained in soil S4 of the present study. The mechanisms for the remarkable increase of rare earth elements in B1, B2 and B3 in soils zones S1, S2 and S3, particularly in soil zones $\mathrm{S} 1$ and S2, can probably be ascribed to the physicochemical and/or biological reactions taking place in the rhizosphere. It is generally recognized that plants can exude low molecular weight organic acids. In the Section 2.2, we pointed out that plant roots remained completely in the soil zone S1, and the mycelium perforated the nylon cloth between S1 and S2. Soil zones $\mathrm{S} 1$ and S2 were mostly affected by plant growth in general, low molecular weight organic acids in particular. Jones [32] reviewed the organic acids in the rhizosphere. Malate, citrate and oxalate all have a high affinity for metal(III) species. This action may be responsible for increase in rare earth elements in fraction B1 in soil zones S1 and S2. Organic acids can also release Mn from manganese oxides through a combination of oxidation and complexation [33]. Organic acids can rapidly release $\mathrm{Fe}$ held in goethite and ferrihydrite, while mobilization from $\mathrm{Fe}_{2} \mathrm{O}_{3}$ and $\mathrm{Fe}_{3} \mathrm{O}_{4}$ is slow [34], therefore, rare earth elements bound $\mathrm{Mn}-\mathrm{Fe}$ oxides were more easily extracted in the present study and can, at least to certain extent, account for the 


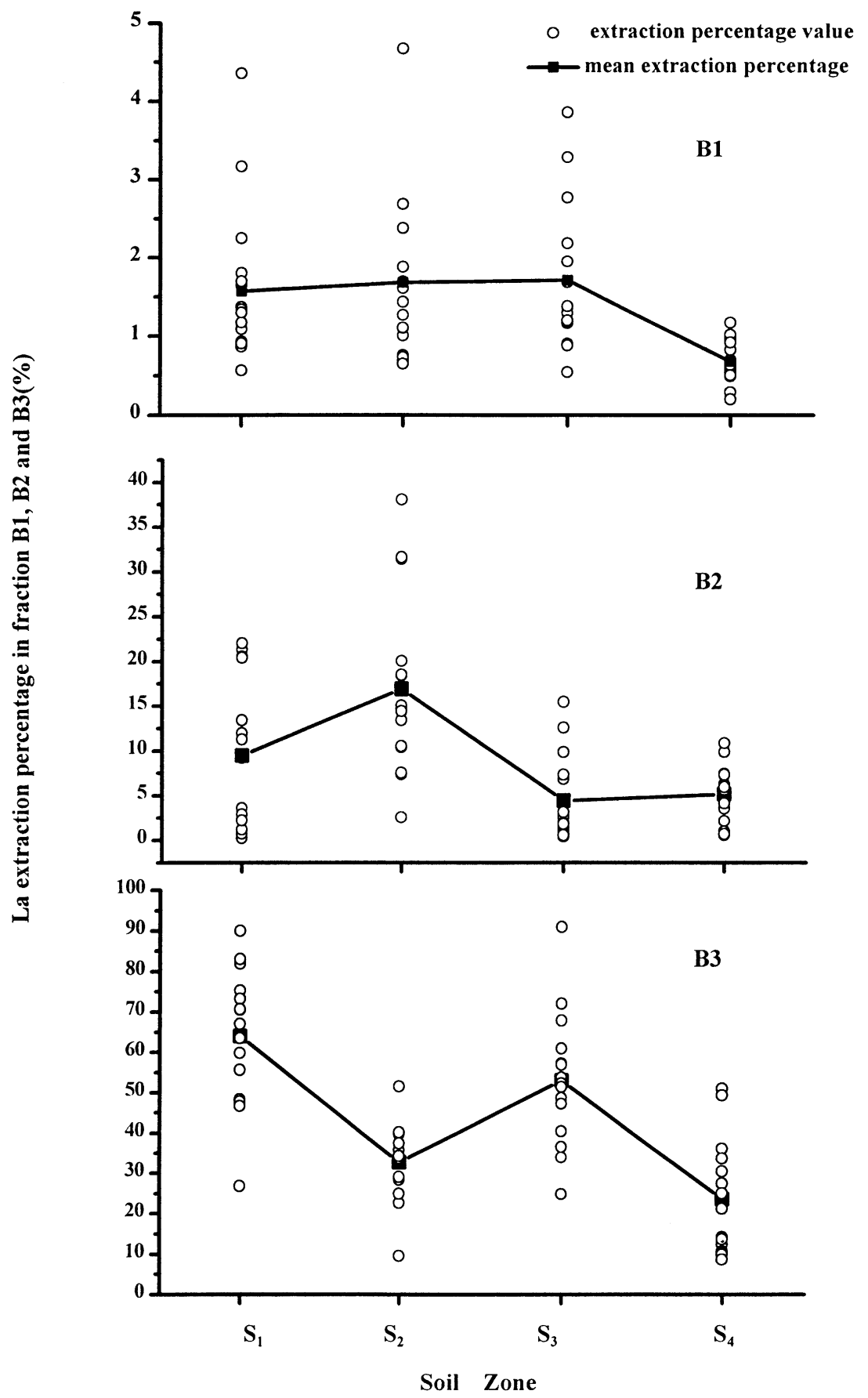

Fig. 3. Distribution of La in fractions B1, B2 and B3 and mean value in different soil zones for 15 soils. 
increase in rare earth elements in B2 in soil zones $\mathrm{S} 1$ and $\mathrm{S} 2$ as well. However, there is no explanation available as to why there is also an increase in rare earth elements in fraction B3 in soil zones S1, S2, and S3 if compared to that in soil S4. It may mean that by the integrated effect of plant growth, the properties of rare earth elements in soils would change, resulting in a substantial change from those phases that cannot be extracted by the three-step procedure into a mobile form.

\subsection{Relationship between speciation and} bioavailability of $\mathrm{La}, \mathrm{Ce}, \mathrm{Pr}$ and $\mathrm{Nd}$ in soils zones S1, S2, S3 and S4

The simple correlation coefficients of rare earth elements in different soil zones and in wheat shoots are listed in Table 4. The correlation coefficients for fraction $\mathrm{B} 1$ in rhizosphere wet soil have better values $\left(r_{\mathrm{La}}=0.830^{* *} ; r_{\mathrm{Ce}}=0.908^{* *} ; r_{\mathrm{Pr}}=\right.$ $\left.0.830^{* *} ; r_{\mathrm{Nd}}=0.854^{* *}\right)$, while the other three soil zones have relatively poor correlation coefficients. These results suggest that the rhizosphere wet soil could be used instead of bulk soil in predicting the availability of rare earth elements in soils.

\section{Conclusions}

Based on the above comparison of the speciation of rare earth elements in soils and their bioavailability between the rhizosphere soil and nonrhizosphere, and between wet soil and air-dried soil, the following conclusions can be drawn:

1. Rhizosphere soil is a small but important environment in soil with quite different physicochemical properties mainly caused by low molecular weight organic acid. The plant availability of rare earth elements in rhizosphere soil was better than that in nonrhizosphere soil.

2. Chemical reactions taking place during the air-drying of the wet soil samples exert a significant effect on the speciation and bioavailability of rare earth elements, resulting in a poorer correlation between the elemental speciation and bioavailability.

3. In order to simulate realistic conditions close to those in the environment it should be recommended to use moist soil samples taken from the rhizosphere for study of speciation analysis and bioavailability of trace elements.

\section{Acknowledgements}

This work was funded by the National Natural Science Foundation of China, the Chinese Academy of Sciences and the Research Center for Eco-Environmental Sciences.

\section{References}

[1] E. Diatloff, C.J. Asher, F.W. Smith, Aust. J. Soil Res. 34 (1996) 735.

[2] S. Zhong, W. Min, in: G. Ni (Ed.), Bioinorganic Chemistry of Rare Earth Elements, Science, Beijing, China, 1995, p. 59.

[3] Y.J. Ji, M.Z. Cui, Y.J. Wang, X.Q. Zhang, in: G.X. Xu, J.M. Xiao (Eds.), New Frontiers in Rare Earth Science and Applications, Vol. 1, Science, Beijing, 1985, p. 700.

[4] G.S. Ding, H.C. Ma, Chinese J. Pharmacol. Sci. 5 (1984) 70.

[5] N.I. Sax, Dangerous Properties of Industrial Materials, Van Nostrand Reinhold, New York, 1984, p. 2358.

[6] E. Sabbioni, R. Pietra, P. Gaglione, G. Vocaturo, F. Colombo, M. Zanoni, F. Rodi, Sci. Total Environ. 26 (1982) 19.

[7] A. Wyttenbach, V. Furrer, P. Schleppi, L. Tobler, Plant Soil 199 (1998) 267.

[8] H. Ichihashi, H. Morita, R. Tastukawa, Environ. Pollut. 76 (1992) 157.

[9] E. Diatloff, C.J. Asher, F.W. Smith, Mater. Sci. Forum Vols. 315-317 (1999) 354.

[10] Q. Tu, X. Wang, L. Tian, L. Dai, Environ. Pollut. 85 (1994) 345.

[11] G.W. Bryan, W.J. Langston, Environ. Pollut. 76 (1992) 89.

[12] A. Tessier, P.G.C. Campbell, M. Bisson, Anal. Chem. 51 (1979) 844.

[13] Ph. Quevauviller, G. Rauret, B. Griepink, Int. J. Environ. Anal. Chem. 51 (1993) 231.

[14] X.Q. Shan, B. Chen, Anal. Chem. 65 (1993) 802.

[15] J.L. Howard, W.J. Van den Brink, Environ. Pollut. 106 (1999) 285.

[16] F.L. Li, X.Q. Shan, T.H. Zhang, S.Z. Zhang, Environ. Pollut. 102 (1998) 269.

[17] H. Sun, X.R. Wang, Q. Wang, L.S. Wang, Chemosphere 36 (1998) 329.

[18] M. Land, B. Ohlander, J. Ingri, J. Thunberg, Chem. Geol. 160 (1999) 121.

[19] E.A. Curl, B. Truelove, The Rhizosphere, Springer, New York, 1986, p. 286.

[20] I. Kraffczyk, G. Trolldenir, H. Beringer, Soil Biol. Biochem. 16 (1984) 316.

[21] J.S. Lao, Handbook for Soil Agriculture and Chemical Analysis, Agriculture Press, Beijing, China, 1988, p. 101, 236 and 386. 
[22] R.A. Youssef, M. Chino, Soil Sci. Plant Nutr. 35 (1989) 609.

[23] S.Z. Zhang, X.Q. Shan, At. Spectrosc. 19 (1997) 140.

[24] S.Z. Zhang, X.Q. Shan, Environ. Pollut. 112 (2001) 395.

[25] C. Kelley, R.E. Mielke, D. Dimaquibo, A.J. Curtis, J.G. Dewitt, Environ. Sci. Technol. 33 (1999) 1439.

[26] L.M. Shuman, Soil Sci. 130 (1980) 336.

[27] M.G. Molloy, R.B. Lockman, Commun. Soil Sci. Plant Anal. 10 (1979) 545.

[28] A. Raveh, Y. Avnomelech, Plant Soil 50 (1978) 545.
[29] F. Rapin, A. Tessier, P.G.C. Campell, R. Carignan, Environ. Sci. Technol. 20 (1986) 836.

[30] R. Bartlett, B. James, Soil Sci. Soc. Am. J. 44 (1980) 721.

[31] F.S. Zhang, Y.P. Cao, Acta Pedol. 29 (1992) 239.

[32] D.L. Jones, Plant Soil 205 (1998) 25.

[33] M.A. Jauregui, H.M. Reisenauer, Soil Sci. Soc. Am. J. 46 (1982) 314.

[34] D.L. Jones, P.R. Darrah, L.V. Kochian, Plant Soil 180 (1996) 57. 